\title{
Cysticercus fasciolaris (Taenia taeniaeformis Larval Stage) in Urban Rats with Illustration of Histopathological Changes in the Liver
}

\author{
Hamid Hasanpour ${ }^{1}$, Faezeh Najafi' ${ }^{1}$, Mohammad Javad Gharagozlou ${ }^{2}$, Sanaz Jafarpour Azami', Arezoo \\ Fadavi $^{1}$, Niloofar Paknezhad ${ }^{1}$, Gholamreza Mowlavi1,3* \\ 1Department of Parasitology and Mycology, School of Public Health, Tehran University of Medical Sciences, Tehran, \\ Iran; ${ }^{2}$ Department of Pathobiology, Faculty of Veterinary Medicine, University of Tehran, Tehran, Iran; ${ }^{3}$ Center for \\ Research of Endemic Parasites of Iran (CREPI), Tehran University of Medical Sciences, Tehran, Iran
}

Received Feb 11, 2018; Accepted Feb 26, 2018

\begin{abstract}
Introduction: Cysticercus fasciolaris is the larval stage of the cestode Taenia taeniaeformis, whose definitive hosts are cats. Rodents and very rarely humans act as intermediate hosts. Here, we investigated the rate of infection among urban rats in the north of Khuzestan province, southwest of Iran, and described the histopathological changes in the liver of infected animals. Methods: One hundred eight rats were collected from the cities Dezful, Sush, and Andimeshk in the north of Khuzestan province during 2014-2015. The rats were sacrificed, dissected, and their livers were removed and examined macroscopically for the presence of the cyst, which were then cut open and inspected for larvae. The cysts of infected livers were examined for the histopathological changes and the recovered larvae were identified based on morphometric features. Results: Out of 108 rats examined, 8 (7.4\%) had small to moderate size cysts. Each cyst had two layers and contained one larva of $C$. fasciolaris. The scolex of the larvae had four suckers and a rostellum armed with two rows of hooks. The infected livers had multiple cysts and liver parenchyma necrosis or neoplastic evidence were not seen in the tissue sections. Conclusion: The small size cysts indicated actue infection, which may explain the absence of sarcoma as well. J Med Microbiol Infect Dis, 2017, 5 (3-4): 43-46. DOI: 10.29252/JoMMID.5.3.4.43
\end{abstract}

Keywords: Cysticercus fasciolaris, Rats, Histopathological changes.

\section{INTRODUCTION}

Cysticercus fasciolaris is the larval stage of Taenia taeniaeformis, a cestode parasite of the small intestine of carnivores, mainly cats [1]. This stage of the helminth is not frequent in humans and was detected just in a post-mortem survey once in India in 1975 [2]. Cysticercus fasciolaris infection has been reported in wild rats worldwide [3-6]. These animals serve as intermediate hosts and acquire infection through the ingestion of contaminated food with Taenia eggs expelled with cat feces [7]. Once ingested, the embryonated eggs hatch in the small intestine and the larvae migrate through the portal blood into the liver, where they form cysts. In rodents, the larvae rarely migrate to other organs such as spleen and lung [8], but cause liver structural changes accompanied by liver cells dysfunction [9]. Also, fibroplasia and progressive inflammation were observed in the liver parenchyma that may progress to fibrosarcoma [3]. Though, the cysts may be seen in different species of rodents, however, due to the host specificity of the parasite at this stage, the development of the larvae of the parasite in rats is more successful [10]. In Iran, a prevalence of $4.34 \%$ was reported in rodents of Kermanshah [11], $0.5 \%$ in Ratus norvegicus rats of Mazandaran province [12], 3.3\% and $1.3 \%$ in the Apodemus sylvaticus and Mus musculus of Hamadan province, respectively [13], and $18.2 \%$ in wild rats of Tehran [14]. Regarding the significant prevalence of $C$. fasciolaris among the rats and the possible accidental transmission to humans, we investigated the rate of infection among rats in the cities of Dezful, Sush, and Andimeshk in the north of Khuzestan province, southwest of Iran during 2014-2015. We also described the histopathological changes in the liver of infected rats.

\section{MATERIAL AND METHODS}

Study area. The study was conducted in urban areas of the north of Khuzestan province, southwest of Iran during 2014-2015. The rats were entrapped in cities of Dezful, Sush, and Andimeshk (Fig.1). The area is semi-arid with hot summers and mild winters with average rainfall and humidity of $384.4 \mathrm{~mm}$ and $46.5 \%$ in year.

Entrapping animals. Live traps baited with bread, cheese, or cucumber were installed in public places, e.g., food and grocery stores at around 8-9 PM and were collected the next day early morning at 6 to $7 \mathrm{AM}$. The entrapment of the rodents was performed during 2014-2015.

\footnotetext{
*Correspondence: Gholamreza Mowlavi

Department of Medical Parasitology and Mycology, School of Public Health, Tehran University of Medical Science, PurSina St., Keshavarz Blvd., Tehran, Iran, 141556446.
}

Email: molavig@yahoo.com

Tel: +98 (21) $88941400 \quad$ Fax: +98 (21) 66462267 


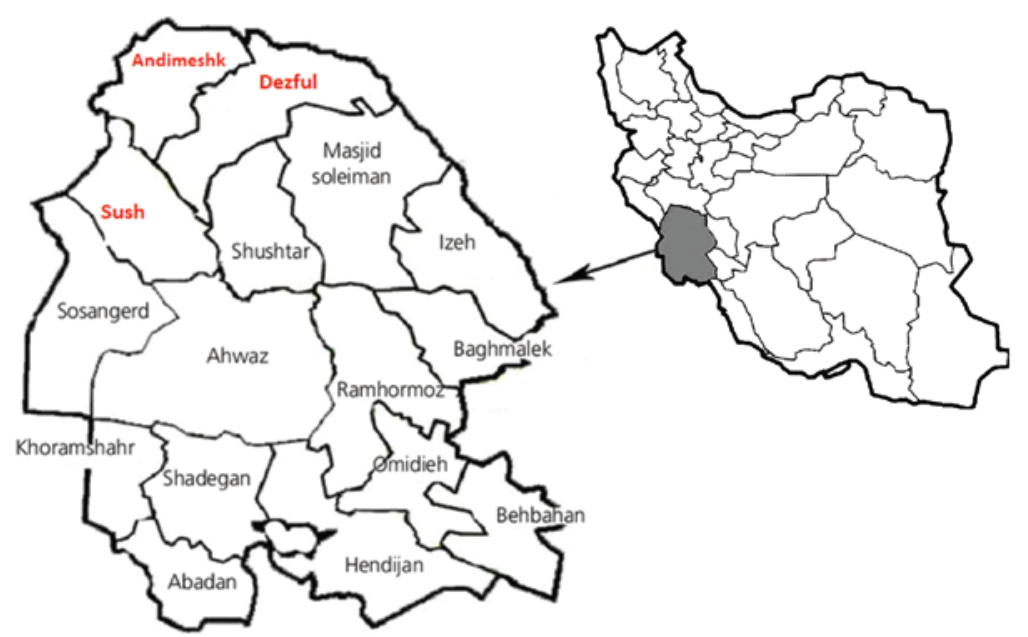

Fig.1. The areas from which the rats were collected in the north of Khuzestan province, Andimeshk, Dezful, and, Sush

Examination of the animals. The rats were sacrificed by chloroform, dissected and their livers were checked macroscopically for cysts. The location, number and size of the cysts in the liver were recorded.

Identification of larvae. The cysts wall were cut open and inspected for larvae. The recovered larvae were stained with lactophenol as described elsewhere [15], and were identified based on morphological and morphometric characters of the hooks as described by others [15]. The small and large hooks were measured, and drawings were made using a microscope equipped with a camera lucida.

Histopathology. The infected tissues with gross lesions were fixed in $10 \%$ neutral buffered formalin and dehydrated in an increasing degree of alcohol, i.e., 70\%, $85 \%, 96 \%$, and $100 \%$ followed by immersion in xylene, each step for one hour. The tissues were embedded in paraffin, and $5 \mu \mathrm{m}$ sections were prepared. The sections were mounted on microscope slides, stained with hematoxylin and eosin and examined under a light microscope with a magnification of 40X [16].

\section{RESULTS}

Animals collection. A total of 108 rats, including $84 R$. norvegicus and 24 Rattus rattus, were collected using live traps from urban areas of the north of Khuzestan province, southwest of Iran.

Gross pathology findings. Out of 108 rats examined, 8 (7.4\%) including seven $R$. norvegicus and one $R$. rattus were infected with larvae. The infected livers had multiple cysts with the size of 1-1.5 mm; most of them were in the right lateral lobe of the liver with the color ranging from white to cream.

Microanatomy findings. The larvae were $4-15 \mathrm{~cm}$ in length with a scolex bearing four large suckers and a rostellum containing two rows of large and small hooks attached to a long neck. There were 34-42 small and large hooks on the rostellum with average size of $224 \pm 12 \mu$ and $381 \pm 18 \mu$ for small and large hooks, respectively. These morphological features matched those of $C$. fasciolaris (Fig. 2).

J Med Microbiol Infect Dis

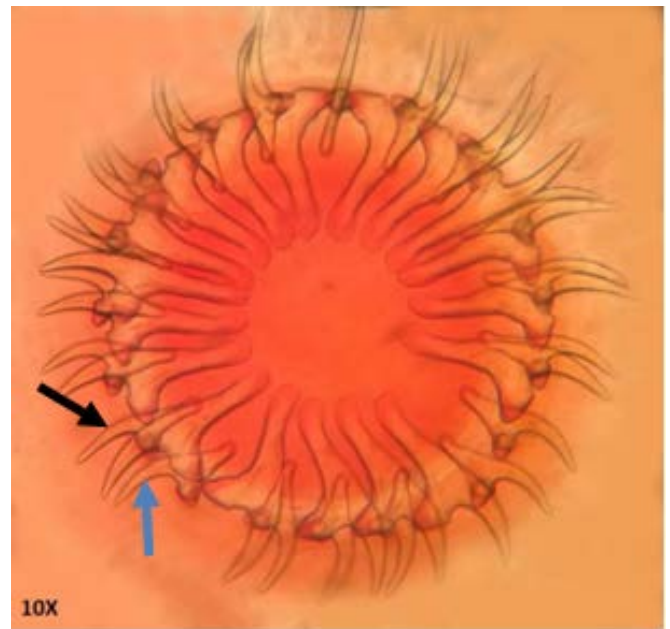

Fig. 2. The $C$. fasciolaris rostellum.

The small and large hooks are indicated by blue and black arrows, respectively.

Histopathology findings. Microscopically, the cysts of C. fasciolaris were partially or wholly embedded in the liver parenchyma or had replaced the tissue (Fig. $3 \mathrm{~A}$ ). The proteinous fluid of each cyst contained one well-developed larva of C. fasciolaris. The scolex of the larva had four suckers, and the outer surface of the parasite was profoundly and evenly pleated (Fig. 3 B). The cyst wall composed of two layers, an inner layer made of loose connective tissue with fibrocytes, active fibroblasts, and cellular matrix delicate connective tissue fibers (Fig. $3 \mathrm{C}$ ). In the internal portion of the inner layer, there were modified connective tissue cells with a single nucleus and a large cytoplasm. These modified cells seem to be the secretory cells of the inner layer of the cyst wall (Fig. $3 \mathrm{D}$ ). The outer layer was composed of an aggregation of lymphocytes, plasmocytes and a very few eosinophil (Fig. 3 E). In two microscopic slides, moderate infiltration of lymphocytes within port spaces of liver parenchyma was evident. Other pathological changes including liver parenchyma necrosis or neoplastic evidence of the liver were not seen in the tissue sections. 

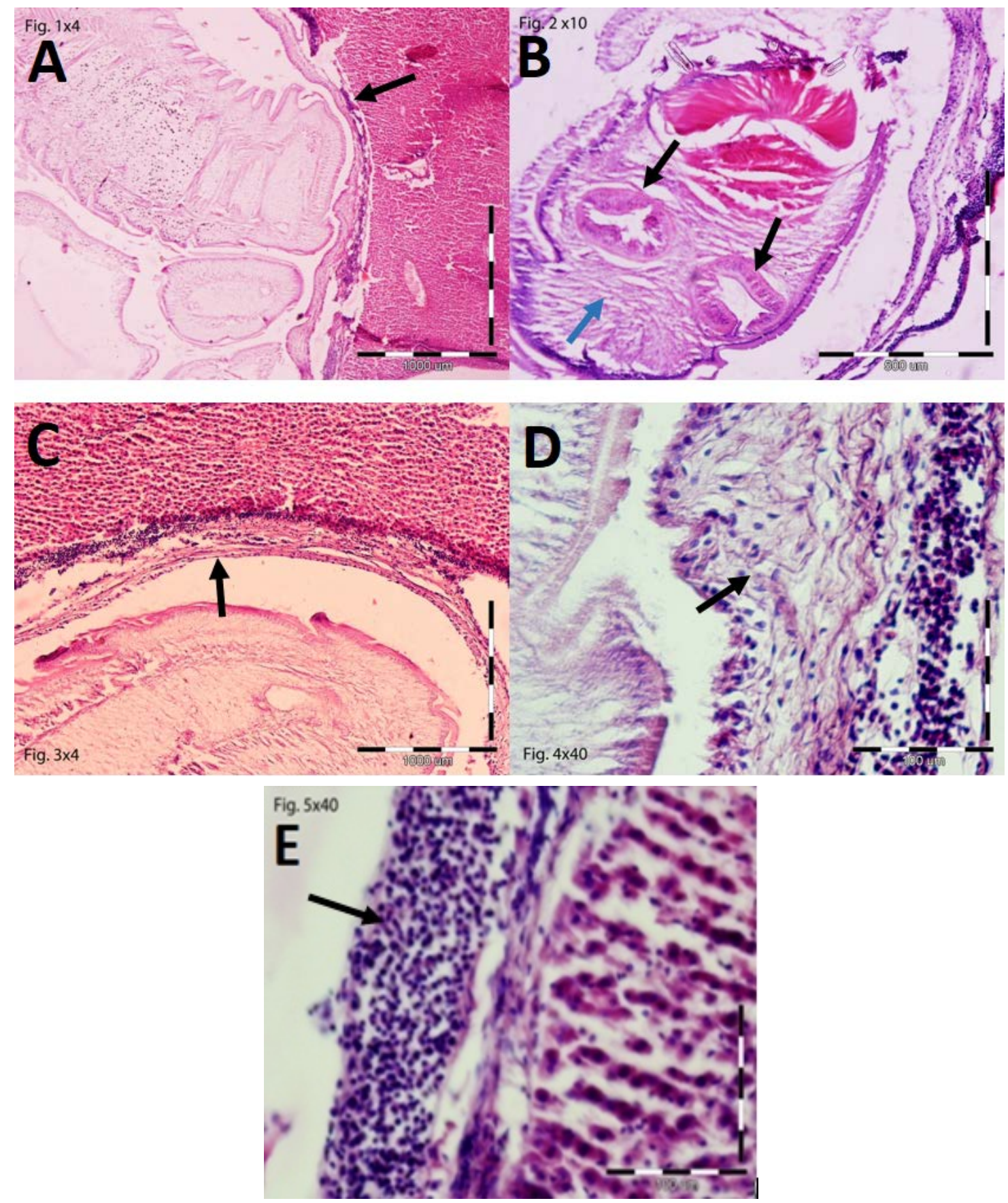

Fig. 3. The liver sections of $C$. fasciolaris-infected rats. (A) the cyst of $C$. fasciolaris (black arrow) in the liver parenchyma; (B) the rostellum (blue arrow) and suckers (black arrows) of the larva; (C) the inner layer (black arrow) of the cyst; (D) the internal portion of the inner layer of the cyst (black arrow); (E) the outer layer of the cyst (black arrow).

\section{DISCUSSION}

In this study, we described $C$. fasciolaris infection in the urban rats from the north of Khuzestan province and demonstrated the histopathological changes of the liver. The infection rate of $C$. fasciolaris in the urban rats was $7.4 \%$. In gross morphological studies, the size of cysts was small to moderate among different rats, which could be attributed to recent infections. Previous studies showed that the size of cysts was related to the duration of the infection [15], and the development stage of the cyst [17]. Moreover, the infected livers had multiple cysts, which may reflect the number of ingested eggs [18]. Other studies have shown the variation in the size of the cysts that might be due to infection at different time intervals [15]. In this study, histopathology of the liver demonstrated a two layer cyst around the $C$. fasciolaris larva, which might be a defense mechanism to limit the growth of the larva [19]. Similar studies have shown two layers of connective tissue around the larva [19]. Also, aggregation of lymphocytes, plasmocytes, and eosinophils in the outer layer of cyst shows the acute nature of infection [15]. Previously, infiltration of mononuclear cells including lymphocytes, 
macrophages, and eosinophils was seen in tissue sections of the liver $[15,19]$. There are reports of hepatic cell necrosis due to the migration of larvae through liver [20]. Some studies have shown the association between implantation of larvae and the development of neoplasia in the liver [10]. The liver sections showed spindle pleomorphic neoplastic cells invading liver parenchyma [9]. Cysticercus fasciolaris act as a foreign body causing chronic irritation and a high degree of inflammatory reactions with neoplasia [9]. Also, hepatic sarcoma has been observed in rats 12-15 months post infection [10]. These findings are in contrast with the result of the present study in which there was no evidence of neoplasia, necrosis, and hepatic sarcoma. Sarcoma occurs in chronic infections which may disseminate in abdominal cavity and pleural [15]. Furthermore, sarcoma is induced by larvae-derived oncogenic substances, microbial infection, and chronic inflammation reactions of the capsule [10]. The tumor development depends on the host species, and laboratory and wild rats are more vulnerable than other rodents [10]. The absence of sarcoma in the present study might be an indication of actue infection since an increase in the post-infection period would increase the chance of hepatic sarcoma.

\section{ACKNOWLEDGEMENT}

This study was supported financially by Tehran University of Medical Sciences, Tehran, Iran.

\section{CONFLICT OF INTEREST}

The authors declare that there are no conflicts of interest associated with this manuscript.

\section{REFERENCES}

1. Hanes MA, Stribling LJ. Fibrosarcomas in two rats arising from hepatic cysts of Cysticercus fasciolaris. Vet Pathol. 1995; 32 (4): 441-4.

2. Sterrba J, Barus V. First record of Strobilocercus fasciolaris (Taenidae-larvae) in man. Folia Parasitol. 1976; 23 (3): 221-6.

3. Yi J-Y, Kim Y-H, Kim H-C, Hahn T-W, Jeong H, Choi C-U, et al. Prevalence of hepatic parasites in Korean wild rats (Rattus norvegicus) and their association with pulmonary arteriolar medial hypertrophy. Vet Pathol. 2010; 47 (2): 292-7.

4. Yi-Fan C, Xu-Heng N, Hui H, Shou-Yang D, Duszynski DW, Jiang-Hui B. Gastrointestinal Parasites of Root Voles, Microtus oeconomus (Rodentia: Muridae), from Haibei Area, Qinghai Province, China. Comp Parasitol. 2014; 81 (2): 185-90.

5. Panti-May J, Hernández-Betancourt S, Rodríguez-Vivas R, Robles MdR. Infection levels of intestinal helminths in two commensal rodent species from rural households in Yucatan, Mexico. J Helminthol. 2015; 89 (1): 42-8.

6. Ito M, Itagaki T. Survey on wild rodents for endoparasites in Iwate Prefecture, Japan. J Vet Med Sci. 2003; 65 (10): 1151-3.
7. Singla L, Singla N, Parshad V, Sandhu B, Singh J. Occurrence and pathomorphological observations of Cysticercus fasciolaris in lesser bandicoot rats in India. Aciar Monogr SER. 2003; 96 : 57-60.

8. Lee B-W, Jeon B-S, Kim H-S, Kim H-C, Yoon B-I. Cysticercus fasciolaris infection in wild rats (Rattus norvegicus) in Korea and formation of cysts by remodeling of collagen fibers. J Vet Diagn Invest. 2016; 28 (3): 263-70.

9.Mahesh Kumar J, Reddy PL, Aparna V, Srinivas G, Nagarajan $\mathrm{P}$, Venkatesan R, et al. Strobilocercus fasciolaris infection with hepatic sarcoma and gastroenteropathy in a Wistar colony. Vet Parasitol. 2006; 141 (3-4): 362-7.

10. Irizarry-Rovira AR, Wolf A, Bolek M. Taenia taeniaeformis-induced metastatic hepatic sarcoma in a pet rat (Rattus norvegicus). J Exot Pet Med. 2007; 16 (1): 45-8.

11. Pakdel N, Naem S, Rezaei F, Chalehchaleh A-A. A survey on helminthic infection in mice (Mus musculus) and rats (Rattus norvegicus and Rattus rattus) in Kermanshah, Iran. Vet Res Forum. 2013; 4(2): 105-9.

12. Gholami SA, Motevali HF, Mobedi I, Shahabi S. Study of helmintic intestinal parasites in the rodents from the rural and central regions of Mazandaran province in the years 1997 to 1999. J Mazandaran Univ Med Sci. 2002; 12 (35): 67-75.

13. Yousefi A, Eslami A, Mobedi I, Rahbari S, Ronaghi H. Helminth infections of house mouse (Mus musculus) and wood mouse (Apodemus sylvaticus) from the suburban areas of Hamadan city, western Iran. Iran J Parasitol. 2014; 9 (4): 511-8.

14. Meshkekar M, Sadraei J, Mahmoodzadeh A, Mobedi I. Helminth infections in Rattus ratus and Rattus norvigicus in Tehran, Iran. Iran J Parasitol. 2014; 9 (4): 548-52.

15. Moudgil AD, Singla LD, Gupta K, Daundkar PS, Vemu B. Histopathological and morphological studies on natural Cysticercus fasciolaris infection in liver of Wistar rats. J Parasit Dis. 2016; 40 (2): 255-8.

16. Singla N, Singla L, Gupta K, Sood N. Pathological alterations in natural cases of Capillaria hepatica infection alone and in concurrence with Cysticercus fasciolaris in Bandicota bengalensis. J Parasit Dis. 2013; 37 (1): 16-20.

17. Sharma R, Tiwari K, Birmingham K, Armstrong E, Montanez A, Guy R, et al. Cysticercus fasciolaris in Brown Rats (Rattus norvegicus) in Grenada, West Indies. J Parasitol Res. 2017; $1-4$.

18. Rodríguez-Vivas R, Panti-May J, Parada-Lopez J, Hernández-Betancourt S, Ruiz-Piña $H$. The occurrence of the larval cestode Cysticercus fasciolaris in rodent populations from the Cuxtal ecological reserve, Yucatan, Mexico. J helminthol. 2011; 85 (4): 458-61.

19. Al-Najjar S, Kadhimand F, Abdalrziak N. Parasitological and Pathological study of the Cysticercus fasciolaris naturally infested white mice. Al Anbar J Vet Sci. 2009; 2: 43-7.

20. Cook R, Trapp A, Williams J. Pathology of Taenia taeniaformis infection in the rat: Hepatic, lymph node and thymic changes. J comp pathol. 1981; 91 (2): 219-26. 